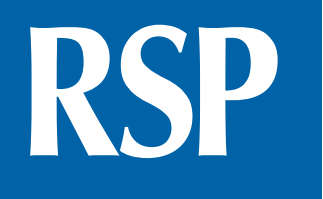

http://www.rsp.fsp.usp.br/
Revista de Saúde Pública

\title{
Recent HIV Infection among men who have sex with men and transgender women in Tijuana
}

\author{
Britt Skaathun' (iD, Heather A. Pines' (iD, Thomas L Patterson" (iD, Shirley J Semple" (iD,

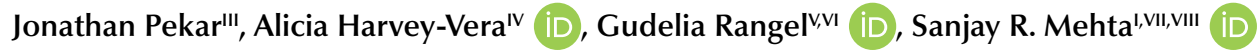 \\ I University of California, San Diego. Department of Medicine. Division of Infectious Diseases and Global \\ Public Health. San Diego, La Jolla, USA \\ " University of California, San Diego. Department of Psychiatry. San Diego, La Jolla, USA \\ III University of California, San Diego. Bioinformatics and Systems Biology Program. San Diego, La Jolla, USA \\ Iv Universidad Xochicalco. Tijuana, Baja California, Mexico \\ $\checkmark$ United States-Mexico Border Health Commission. Tijuana, Baja California, Mexico \\ vı El Colegio de la Frontera Norte. Tijuana, Baja California, Mexico \\ VII University of California, San Diego. Department of Pathology. San Diego, La Jolla, USA \\ VIII San Diego Veterans Affairs Medical Center. Department of Medicine. San Diego, USA
}

\section{ABSTRACT}

OBJECTIVE: To characterize recent HIV infections among newly diagnosed men who have sex with men and transgender women in Tijuana.

METHODS: Limiting Antigen (LAg)-Avidity testing was performed to detect recent HIV infection within a cohort of newly-diagnosed men who have sex with men and transgender women in Tijuana. Logistic regression was used to determine characteristics associated with recent infection. A partial transmission network was inferred using HIV-1 pol sequences. Tamura-Nei 93 genetic distances were measured between all pairs of sequences, and the network was constructed by inferring putative transmission links (genetic distances $\leq 1.5 \%$ ). We assessed whether recent infection was associated with clustering within the inferred network.

Correspondence:

Britt Skaathun

9500 Gilman Drive 0507

92093-0507 La Jolla, CA, USA

E-mail Bskaathun@health.ucsd.edu

Received: Nov 2, 2019

Approved: Fev 18, 2020

How to cite: Skaathun $B$, Pines HA, Patterson TL, Semple SJ, Pekar K, Harvey-Vera A, et al. Recent HIV Infection among men who have sex with men and transgender women in Tijuana. Rev Saude Publica. 2020;54:82.

Copyright: This is an open-access article distributed under the terms of the Creative Commons Attribution License, which permits unrestricted use, distribution, and reproduction in any medium, provided that the original author and source are credited.
RESULTS: Recent infection was detected in 11\% (22/194) of newly-diagnosed participants. Out of the participants with sequence data, $60 \%$ (9/15) with recent infection clustered compared with $31 \%$ (43/139) with chronic infection. Two recent infections belonged to the same cluster. In adjusted analyses, recent infection was associated with years of residence in Tijuana $(\mathrm{OR}=1.5$; 95\%CI 1.01-1.09), cocaine use (past month) (OR = 8.50; 95\%CI 1.99-28.17), and ever experiencing sexual abuse $(\mathrm{OR}=2.85$; 95\% CI 1.03-7.85).

DISCUSSION: A total of $11 \%$ of men newly diagnosed with HIV who have sex with men and transgender women in Tijuana were recently infected. The general lack of clustering between participants with recent infection suggests continued onward HIV transmission rather than an outbreak within a particular cluster.

DESCRIPTORS: HIV Infections, epidemiology. Sexual and Gender Minorities. Transgender Persons. Disease Transmission, Infectious. 


\section{INTRODUCTION}

Individuals who are in the primary stages of infection are responsible for a disproportionate number of new infections through several factors, including higher viral loads and being unaware of their status ${ }^{1,2}$. Rapid and targeted delivery of ART to people with recent infection may be more cost-effective at reducing transmission in resource-constrained settings than uniform delivery to similar numbers of persons living with HIV due to the increased probability of transmission during recent infection ${ }^{3,4}$. A study of men who have sex with men (MSM) with recent HIV infection in San Diego found that $18 \%$ of putative transmission links occurred during the putative source's period of recency ${ }^{5}$. Similarly, a risk network intervention that located people with recent infection, found that the networks of those with recent infection were more likely to include others with recent infection than those with chronic HIV infections ${ }^{6}$.

Accurate estimates of HIV incidence among MSM and transgender women (TW) in Tijuana, Baja California, Mexico, are unavailable despite being the most heavily affected risk population in the area (HIV prevalence: $\mathrm{MSM} / \mathrm{TW}=20 \%$, people who inject drugs $[\mathrm{PWID}]=4 \%$, female sex workers $[\mathrm{FSW}]=6 \%)^{7}$. In the past 25 years, there have been only three estimates of HIV prevalence among MSM in Tijuana, and no reports of HIV incidence. Available estimates suggest that HIV prevalence has increased over time from $11 \%$ in a 1991 convenience sample of $\mathrm{MSM}^{8}$, to $18.9 \%$ in a 2002 convenience sample of $\mathrm{MSM}^{9}$, and ultimately $20 \%$ (95\% CI 12.5-29.1) in a 2014 sample of MSM and TW recruited by respondent-driven sampling $(\mathrm{RDS})^{7}$. Whereas these estimates are not sufficient to establish a trend considering the disparate and non-probabilistic sampling techniques applied, they are all notably high and have remained high over the past few decades. Reducing new transmissions is a key goal in the public health response to HIV ${ }^{10}$.

Preventive interventions can be directed towards the uninfected as well as the infected population, treatment as prevention (TasP) has reduced incident infections in HIV serodiscordant couples $^{11,12}$. However, despite universal access to HIV care in Mexico, knowledge of one's HIV status and subsequent engagement in HIV care are low ${ }^{7}$. The study of MSM and TW in 2014 found that out of those who tested positive, only $11 \%$ were previously aware of their HIV status. ${ }^{7}$ Estimates of ART use among MSM and TW are not available? ${ }^{7}$ However, in a pooled sample of data from multiple studies assessing PWID, FSW, and MSM, the prevalence of ART use was only $3.7 \%$ overall, indicating that the rate among MSM is also quite low ${ }^{13}$. High rates of undiagnosed infection and subsequent low rates of linkage to care among MSM in Tijuana hinder successful treatment, leading to continued transmission. Since Mexico is a resource-limited country, it may be unrealistic to target all MSM and TW who are potentially out-of-care. Therefore, this study aims to estimate and characterize recent HIV infections among MSM and TW in Tijuana, which can serve as a starting point for effective resource allocation.

\section{METHODS}

\section{Study Population}

Data come from Proyecto Enlaces (Links Project), which was designed to compare the effectiveness of partner contact tracing (PCT) (changed to respondent-driven sampling [RDS] after 9 months) and venue-based sampling (VBS) for identifying undiagnosed infection among MSM and TW in Tijuana (as described elsewhere) ${ }^{14}$. PCT (eliciting and contacting sexual partners of participants who were recently diagnosed with HIV) was used between March and December of $2015^{15}$. RDS, a chain-referral sampling technique that modifies snowball sampling by limiting the number of individuals a participant can recruit, was used between January 2016 and November 2018. VBS was conducted between March 2015 and November 2018 at 36 venues identified via formative research as locations attended by MSM 
and TW to meet sexual partners (e.g., nightclubs, bars, public spaces, motels). Individuals identified via VBS were eligible for HIV testing if they were at least 18 years old, cisgender male or transgender female, reported anal sex with a cisgender male or transgender female in the past four months, and they did not report a previous HIV diagnosis.

Eligibility criteria for RDS seeds included: 1.) Persons assigned male sex at birth identifying as cisgender male or transgender female, 2.) $\geq 18$ years of age, 3.) Anal sex with a cisgender male or transgender female in the past 4 months, 4.) Tijuana resident, and 5.) Knowing $\geq 15$ MSM or TW who are $\geq 18$ years old in Tijuana (changed to 5 MSM or TW in April 2017 to boost RDS recruitment). RDS seeds received three vouchers (changed to six vouchers to boost RDS recruitment in January 2018) to recruit MSM or TW peers from their social networks to the study. Peer-recruits were then eligible to recruit three to six of their peers if they were at least 18 years old, cisgender male or transgender female, and reported anal sex with a cisgender male or transgender female in the past 12 months. Those who did not report a previous HIV diagnosis were also eligible for HIV testing. RDS was initiated by 33 seeds who were identified by VBS or referrals from Tijuana's municipal HIV clinic, and they were selected to be diverse regarding HIV status, age, socio-economic status, sexual orientation, gender identity, and recruitment venue.

Individuals could be identified multiple times by the same or a different recruitment method. Those identified more than once who remained eligible for HIV testing were re-tested if it had been at least three months since their last test. Eligible individuals identified via VBS underwent rapid HIV testing at recruitment venues or at the study site if they preferred, whereas those identified via RDS underwent rapid HIV testing at the study site.

Participants answered a set of questionnaires designed to collect socio-demographic, behavioral, psychosocial, and socio-contextual data, as well as detailed data on up to 20 of their sexual partners in the past four months. Each participant (regardless of recruitment method) received 150 Mexican (MXN) pesos for HIV testing and 450 MXN pesos for completing the study questionnaire (equivalent to approximately 8 and 24 US dollars), and those recruited with RDS received an additional $100 \mathrm{MXN}$ pesos (approximately 5 US dollars) for each successful recruit enrolled into the study. All participants signed a written informed consent and research methods were reviewed and approved by the Institutional Review Boards at the University of California, San Diego and Xochicalco University in Tijuana, Mexico. All procedures were conducted in accordance with the 1964 Helsinki Declaration and its amendments.

\section{Laboratory Testing}

All participants provided blood samples for serologic testing and HIV sequencing. Rapid test-positive individuals provided an additional blood sample for confirmatory testing via immunofluorescence assay at the San Diego County Public Health Laboratory and were offered enrollment in Proyecto Enlaces. Confirmatory HIV test results were delivered to rapid test-positive individuals within two weeks and those confirmed HIV-positive were referred for free HIV care at Tijuana's municipal HIV clinic. HIV-1 Limiting Antigen (LAg) Avidity testing (Sedia Biosciences, Portland, OR) was performed on blood samples provided by newly-diagnosed participants, including those diagnosed as part of this study or in the past two months for PCT index participants and RDS seeds, to distinguish recent HIV-1 infections from chronic infections with an estimated cut-off of around 130 days $^{16}$. DNA was extracted from stabilized blood using DNAGard (Biomatrica, San Diego, CA). Polymerase chain reaction (PCR) and Sanger sequencing were used to obtain HIV-1 partial pol sequences from the extracted DNA. To maximize our ability to obtain a HIV sequence from these participants, we chose partial pol sequence from bp $2726 \rightarrow 3214^{17-19}$.

\section{Measures}

The outcome of interest was recent HIV infection determined by LAg-Avidity testing. Exposures of interest are described below, and they were collected via questionnaires 
administered using computer-assisted personal-interviewing (CAPI) technology by local, Spanish-speaking interviewers with experience working with sexual and gender minorities and who were also trained in non-judgmental interviewing techniques to encourage an open and honest reporting on sensitive information.

\section{Socio-Demographic Factors}

This section included age, gender identity (cisgender male or transgender female), sexual orientation (homosexual/gay, bisexual, heterosexual/straight, or questioning), highest level of education (cannot read or write, some grade school, grade school, some secondary school, secondary school, some high school, high school, some college, college or advanced degree), employment status (unemployed, part-time or full-time).

\section{Substance Use and Sexual Behaviors}

The following characteristics were measured: Substance use measures elicited information on lifetime and past month use of illicit drugs (marijuana, heroin, inhalants, methamphetamine, ecstasy, cocaine, tranquilizers, barbiturates, amyl nitrites (poppers), $\gamma$-hydroxybutyric acid (GHB), ketamine and other). Sexual behaviors were derived from a sexual network survey, which asked about up to 20 anal or vaginal sex partners in the past four months ${ }^{20}$.

\section{Socio-Structural Factors}

This section included the following: length of time living in Tijuana (years), deportation from the United States (US), homelessness (in previous four months), and incarceration history (lifetime). History of abuse was assessed via three separate questions about whether participants had ever been forced or coerced to have sex against their will, physically abused (i.e., hit or assaulted), or emotionally abused, and whether they had experienced abuse in the previous 4 months. Internalized stigma related to having sex with men was measured among MSM participants using a 9-item scale ${ }^{21}$. Participants indicated their level of agreement with the scale items via Likert scale responses $(1=$ strongly disagree, 2 = disagree, 3 = neither agree nor disagree, $4=$ agree, $5=$ strongly agree). We adapted the nine items presented to MSM to reflect experiences of TW (e.g., "I have tried to stop being attracted by men in general" was changed to "I have tried to stop identifying myself as a woman in general") (Cronbach's alpha $=0.89$ for adapted items), to measure internalized stigma related to gender identity among TW participants. Participants' responses to items measuring internalized stigma were summed to create a score, with higher scores indicating greater levels of internalized stigma. Outness about having sex with men or being a transgender woman was assessed by asking participants to describe how 'out' they are about having sex with men or being a transgender woman on a scale of 1-7 ( 1 = not out to anyone; $4=$ out to about half the people I know; 7 = out to everyone $)^{22}$. Social support was measured via Likert scale responses $(1=$ strongly disagree, 2 = disagree, 3 = agree, 4 = strongly agree) with 7 items on help and support received from friends and family (e.g., "Do people close to you let you know they care about you?"). Social support scores were computed by averaging responses across items and transforming the result average to a score on a 100-point scale, with higher total scores indicating greater social support ${ }^{23}$.

\section{Inference of HIV Transmission Network}

HIV-1 pol sequences were aligned using MUSCLE in AliView ${ }^{24}$ and analyzed to infer an HIV transmission network using HIV TRAnsmission Cluster Engine (HIV-TRACE) ${ }^{25}$. After aligning sequences to an HXB2 reference sequence, Tamura-Nei 93 (TN93) genetic distances were measured between all pairs of sequences. To construct the HIV transmission network, participants whose sequences had TN93 genetic distances $\leq 0.015$ substitutions per site were connected by putative HIV transmission links. This genetic distance threshold has been validated to identify partners with direct or indirect epidemiological links ${ }^{26}$, and it 
is used for molecular HIV surveillance in U.S. public health ${ }^{27}$. It is a widely used threshold for non-outbreak related research ${ }^{28-32}$. Clustering was defined as having $\geq 1$ putative transmission link within the inferred network.

\section{Statistical Analysis}

Unadjusted and adjusted logistic regression models were used to assess the relationship between recent HIV infection and participant's characteristics across the following domains: 1. Socio-demographics, 2. Socio-structural factors, and 3. Substance-use and sexual behaviors. Characteristics with large magnitudes of association in unadjusted models were considered as exposures of interest in separate adjusted models to estimate the total effect of each exposure of interest on recent HIV infection ${ }^{33}$. Potential confounding variables were selected for inclusion in adjusted models based on a priori knowledge about their interrelationships with the exposures of interest and recent HIV

Table 1. Estimated number and characteristics, and unadjusted logistic regression of factors associated with recent HIV infection among MSM/TW in the Enlaces Cohort, Tijuana, Baja California, Mexico $(N=195)$.

\begin{tabular}{|c|c|c|c|c|}
\hline Characteristic & $\begin{array}{c}\text { Incident infection } \\
\mathrm{n}=\mathbf{2 2} \\
\mathrm{n}(\%)\end{array}$ & $\begin{array}{c}\text { Chronic infection } \\
n=172 \\
n(\%)\end{array}$ & OR & $95 \% \mathrm{Cl}$ \\
\hline Transgender woman & $1(6)$ & $18(11)$ & 2.15 & $0.27-17.17$ \\
\hline Age, years (median, IQR) & $33(23-44)$ & $31(26-39)$ & 0.99 & $0.95-1.05$ \\
\hline Education $>$ high school & $12(57)$ & $81(49)$ & 1.40 & $0.56-3.50$ \\
\hline Unemployment & $8(38)$ & $62(37)$ & 0.97 & $0.38-2.47$ \\
\hline Time of residence in Tijuana, years (median, IQR) & $19(9-23)$ & $8(2-18)$ & 1.05 & $1.01-1.09$ \\
\hline \multicolumn{5}{|l|}{ Sexual orientation } \\
\hline Gay/homosexual & $11(52)$ & $94(57)$ & - & - \\
\hline Straight/heterosexual & 0 & $19(11)$ & - & - \\
\hline Bisexual & $8(28)$ & $52(31)$ & - & - \\
\hline Questioning & $2(9)$ & $1(<1)$ & - & - \\
\hline Ever deported from the U.S. & $3(14)$ & $56(34)$ & - & - \\
\hline Homeless (past four months) & $1(6)$ & $26(16)$ & 0.31 & $0.04-2.40$ \\
\hline Ever incarcerated & $3(14)$ & $51(31)$ & 0.38 & $0.11-1.34$ \\
\hline Number of sexual partners (median, IQR) & $3(1.5-4.5)$ & $4(1-10)$ & 0.98 & $0.94-1.02$ \\
\hline \multicolumn{5}{|l|}{ Substance use (past month) } \\
\hline Marijuana & $10(48)$ & $51(31)$ & 2.05 & $0.82-5.13$ \\
\hline Cocaine & $5(24)$ & $8(5)$ & 6.21 & $1.82-21.25$ \\
\hline Methamphetamines & $4(19)$ & $61(37)$ & 0.41 & $0.13-1.27$ \\
\hline Amyl nitrate (poppers) & $4(19)$ & $17(10)$ & 2.08 & $0.63-6.89$ \\
\hline Intravenous drugs & $1(5)$ & $20(12)$ & 0.37 & $0.05-2.89$ \\
\hline Ever experienced sexual abuse & $11(61)$ & $58(36)$ & 2.76 & $1.02-7.52$ \\
\hline Ever experienced physical abuse & $6(33)$ & $78(49)$ & 0.53 & $0.19-1.49$ \\
\hline Internalized stigma related to being MSM/TW (median, IQR) & $25(18-34)$ & $20(17-30)$ & 1.04 & $0.99-1.10$ \\
\hline Outness scale (median, IQR) & $4.5(1-7)$ & $5(3-7)$ & 0.91 & $0.75-1.11$ \\
\hline Social support scale (median, IQR) & $75(52-92)$ & $59(34-88)$ & 1.01 & $0.99-1.03$ \\
\hline \multicolumn{5}{|l|}{ Recruitment method } \\
\hline РCT & $3(14)$ & $19(11)$ & 1.38 & $0.36-5.36$ \\
\hline RDS & $7(32)$ & $48(28)$ & 1.28 & $0.47-3.44$ \\
\hline VB & $12(54)$ & $105(61)$ & Ref. & \\
\hline Clustered in transmission network & $9(60)$ & $43(31)$ & 3.35 & $1.12-9.99$ \\
\hline
\end{tabular}

OR: odds ratio; 95\% Cl: 95\% confidence interval; IQR: interquartile range; PCT: partner contact tracing; RDS: respondent driven sampling,

VB: venue based.

Note: significant values are shown in bold. 
infection. All regression analyses were conducted using Stata version 15. Assortativity was estimated within the inferred HIV transmission network regarding recent infection (i.e., the tendency for participants with recent infection to have putative transmission links to other participants with recent infection) to assess whether recent infections might be the result of an outbreak or ongoing transmission ${ }^{34}$. To assess the statistical significance of observed assortativity patterns within the inferred transmission network considering the relative representation of the duration of HIV infection (recent vs. chronic) in these clusters, we generated expected distributions for the assortativity parameter by randomly permuting the duration of HIV infection labels on the static network 5000 times using Python 3.6.8 and SciPy. 1.3.1.

\section{RESULTS}

In total, 232 HIV-positive MSM and TW were enrolled in Proyecto Enlaces. Out of which, 203 were newly diagnosed HIV-positive (188 as part of the study) and 175 had HIV-1 pol sequence data (sequences could not be generated from blood samples provided by 36 participants). Out of newly-diagnosed participants, 198 received the LAg Avidity testing and 155 had both sequences and LAg results. The median age of the participants with LAg test results $(\mathrm{n}=195)$ was 31 (IQR 24-39). Most participants identified themselves as male (90\%), were employed (62\%), and were stably housed in the previous 4 months (85\%). Half of the sample reported having at least high school education (50\%), and just over a quarter reported a history of incarceration (28\%). Most participants reported identifying as gay/homosexual (57\%), while $32 \%$ identified as bisexual, $10 \%$ identified as straight/heterosexual, and $2 \%$ were questioning. Out of those who identified themselves as straight/heterosexual, $58 \%$ identified as transgender. The median length of time lived in Tijuana was 9 years (IQR 3-19). Most participants were born in Mexico (95\%), with only $32 \%$ born in the state of Baja California. Thirty-one percent reported ever being deported from the US. The most commonly reported drugs used in the previous month were methamphetamine (34\%) and marijuana (32\%). Eleven percent reported injecting drugs in the past month, and $61 \%$ reported using drugs before sex in the past 4 months. The median number of sexual partners in the previous 4 months was 4 (IQR 1.5-9). Regarding socio-structural factors, $48 \%$ reported ever experiencing physical abuse and $39 \%$ reported ever experiencing sexual abuse. The median scores on the internalized stigma scale, outness scale, and social support scale were 21 (interquartile range [IQR] 17-30), 5 (IQR 2-7), and 66 (IQR 38-88), respectively.

Recent infection was detected in 11\% (22/194) of newly-diagnosed participants. A breakdown of select characteristics by the duration of HIV infection (recent vs. chronic) can be found in Table 1. In adjusted analyses, recent infection was associated with length of time living in Tijuana (OR $=1.05$ per year; $95 \% \mathrm{CI} 1.01-1.09)$, cocaine use in the previous month ( $\mathrm{OR}=7.50$; 95\%CI 1.99-28.17), and ever having experienced sexual abuse ( $\mathrm{OR}=2.85$; 95\%CI 1.03-7.85) (Table 2). The results were not sensitive to type of recruitment.

Of the 154 newly diagnosed participants with available sequence and LAg data, 60\% (9/15) of those with recent infection clustered compared with $31 \%$ (43/139) of those with chronic infection. Figure 1 represents a network of potential transmission partners: participants

Table 2. Adjusted Logistic Regressions: Factors Associated with Recent HIV Infection among MSM and TW among the Enlaces Cohort, Tijuana, Baja California, Mexico $(\mathrm{N}=187)$.

\begin{tabular}{lccc}
\hline & OR & $\mathbf{9 5} \% \mathbf{C I}$ & p \\
\hline Years living in Tijuana & 1.05 & $1.01-1.09$ & 0.03 \\
Cocaine use (past month) & 7.50 & $1.99-28.17$ & 0.003 \\
Ever experienced sexual abuse & 2.85 & $1.03-7.85$ & 0.04 \\
\hline
\end{tabular}

OR: odds ratio; $95 \% \mathrm{Cl}$ : 95\% confidence interval

Note: Models adjusted for age and education 
whose sequences had genetic distances $\leq 0.015$ substitutions per site connected by putative HIV transmission links. As depicted by the figure, there were 19 clusters and the maximum cluster size was 15 persons. Two recent infections belonged to the same cluster, but the rest were in separate clusters. The assortativity coefficient was -0.168 (two-tailed, $p=0.13$ ). Figure 2 depicts the results of the permuted distribution compared to the observed assortativity coefficient. Each permutation retained the original network structure, but randomly assigned the recent infections throughout the network. The two-tailed p-value of 0.13 reveals that the null hypothesis (recent infections do not cluster together) cannot be rejected.

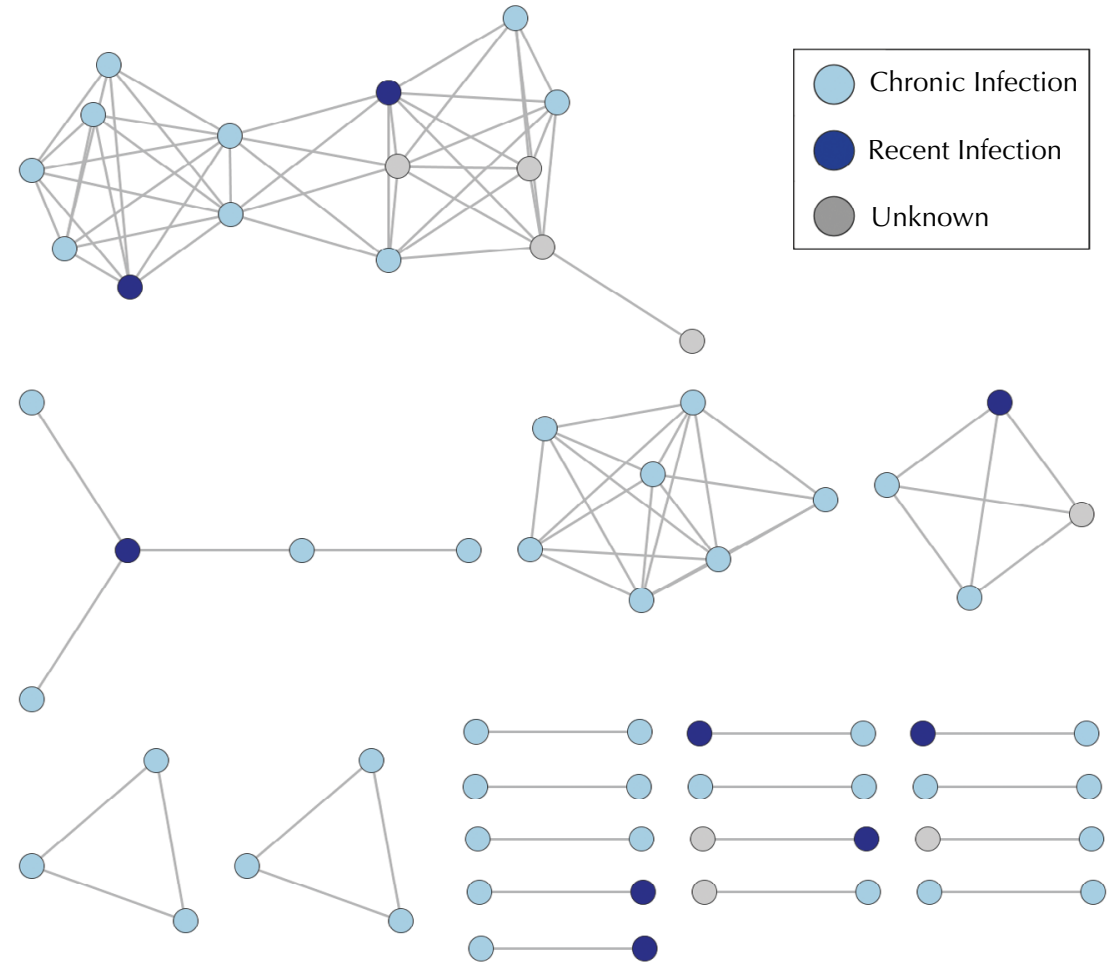

Figure 1. Location of recent HIV infections within the HIV transmission network.

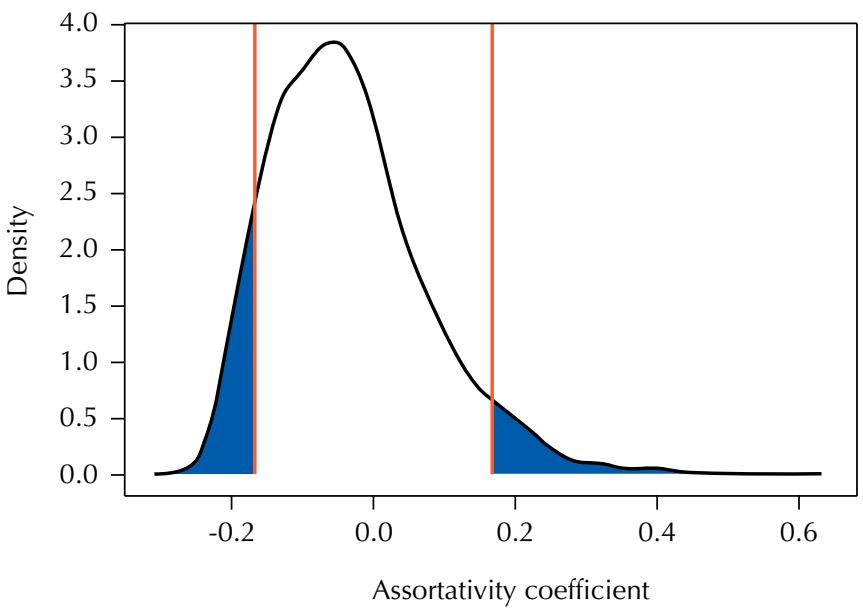

Figure 2. Comparison of observed assortativity coefficient and assortativity coefficients computed from 5000 randomly generated networks (reference line indicates observed assortativity coefficient). 


\section{DISCUSSION}

We found that $11 \%$ of MSM and TW who were newly diagnosed with HIV likely acquired it within 130 days of their HIV test (i.e. were recently infected with HIV). Recent HIV infection was associated with the length of time living in Tijuana and cocaine use in the past month. Considering the low proportion of participants who were born in Baja California (32\%), this finding may indicate that MSM and TW from other areas of Mexico migrate to Tijuana for economic opportunities, and Tijuana also provides more opportunities to test for HIV ${ }^{35}$. As such, Tijuana may provide more access to HIV prevention and care services than more rural areas, increasing HIV testing rates and consequently our ability to detect recent infections among those who have lived in the area for longer periods.

Furthermore, we found an association between recent HIV infection and ever experiencing sexual abuse. This finding is congruent with previous literature showing that sexual abuse has been associated with increased number of sexual partners, condomless anal intercourse, condomless anal intercourse with a HIV serodiscordant partner, and it is commonly reported by Latino MSM than White MSM in the U.S. ${ }^{36,37}$. The mechanism behind this association is not well understood as mental health indicators such as depression and anxiety have not been found to mediate the association ${ }^{38}$. Interestingly, MSM and TW in Tijuana with a history of sexual abuse were also more likely to have been tested for HIV in their lifetime compared to those who have not experienced sexual abuse ${ }^{38}$.

The low magnitude of the estimated assortativity coefficient reveals that, in our sample, recent infections were not likely to cluster together within the inferred HIV transmission network. This probably suggests that a continued onward HIV spread occurs along multiple different transmission chains among MSM and TW population in Tijuana, rather than an outbreak within a particular cluster. These results also suggest that chronically infected individuals may be contributing to new infections in this population.

Our study is limited in its ability to infer causality between participants' characteristics and recent HIV acquisition due to the cross-sectional nature of the study design. Moreover, the sample size was relatively small, and consequently the HIV transmission network is under sampled. Despite these limitations, this study serves as evidence of substantial ongoing transmission within the population, which calls for the need of a population-based HIV incidence estimate in the region.

\section{CONCLUSIONS}

This study identified that $11 \%$ of newly HIV diagnosed MSM and TW in Tijuana were recently infected with HIV. The lack of clustering between the recent infections suggests continued onward HIV transmission among MSM and TW population rather than an outbreak within a particular cluster. Considering the large proportion of HIV-positive MSM and TW in Tijuana who are unaware of their infection, our results suggest a broad, active, and relatively undefined HIV epidemic among these populations in Tijuana. Considering the resource-constrained context, results indicate that resources should be allocated to those with characteristics associated with recent infection (longer duration in Tijuana, current cocaine use, and history of sexual abuse) as they are most likely to be recently infected. This study also highlights the need to obtain a high-quality estimate of HIV incidence across Tijuana to monitor the epidemic and track the impact of interventions.

\section{REFERENCES}

1. Attia S, Egger M, Muller MF, Zwahlen M, Low N. Sexual transmission of HIV according to viral load and antiretroviral therapy: systematic review and meta-analysis. AIDS. 2009;23(11):1397-404. https://doi.org/10.1097/QAD.0b013e32832b7dca 
2. Quinn TC, Wawer MJ, Sewankambo N, Serwadda D, Li C, Wabwire-Mangen F, et al. Viral load and heterosexual transmission of Human Immunodeficiency Virus Type 1. Rakai Project Study Group. N Engl J Med. 2000;342(13):921-9. https://doi.org/10.1056/NEJM200003303421303

3. Wawer MJ, Gray RH, Sewankambo NK, Serwadda D, Li X, Laeyendecker, et al. Rates of HIV-1 transmission per coital act, by stage of HIV-1 infection, in Rakai, Uganda. J Infect Dis. 2005;191(9):1403-9. https://doi.org/10.1086/429411

4. Hollingsworth TD, Anderson RM, Fraser C. HIV-1 transmission, by stage of infection. J Infect Dis. 2008;198(5):687-93. https://doi.org/10.1086/590501

5. Pines HA, Wertheim JO, Liu L, Garfein RS, Little SJ, Karris MY. Concurrency and HIV transmission network characteristics among men who have sex with men with recent HIV. AIDS. 2016;30(18):2875-83. https://doi.org/10.1097/QAD.0000000000001256

6. Nikolopoulos GK, Pavlitina E, Muth SQ, Schneider J, Psichogiou M, Williams LD, et al. A network intervention that locates and intervenes with recently HIV-infected persons: The Transmission Reduction Intervention Project (TRIP). Sci Rep. 2016;6:38100. https://doi.org/10.1038/srep38100

7. Pitpitan EV, Goodman-Meza D, Burgos JL, Abramovitz D, Chavarin CV, Torres K, et al. Prevalence and correlates of HIV among men who have sex with men in Tijuana, Mexico. J Int AIDS Soc. 2015;18(1):19304. https://doi.org/10.7448/IAS.18.1.19304

8. Güereña-Burgueño F, Benenson AS, Sepúlveda Amor J. HIV-1 prevalence in selected Tijuana sub-populations. Am J Public Health. 1991;81(5):623-5. https://doi.org/10.2105/ajph.81.5.623

9. Rajasingham R, Mimiaga MJ, White JM, Pinkston MM, Baden RP, Mitty JA. A systematic review of behavioral and treatment outcome studies among HIV-infected men who have sex with men who abuse crystal methamphetamine. AIDS Patient Care STDS. 2012;26(1):36-52. https://doi.org/10.1089/apc.2011.0153

10. United Nations Joint Programme on HIV/AIDS. 90-90-90: an ambitious treatment target to help end the AIDS epidemic. Geneva: UNAIDS; 2014 [cited 2018 Oct 6]. Available from: http://www.unaids.org/sites/default/files/media_asset/90-90-90_en.pdf

11. Cohen MS, Chen YQ, McCauley M, Gamble T, Hosseinipour MC, Kumarasamy N, et al. Prevention of HIV-1 infection with early antiretroviral therapy. N Engl J Med. 2011;365(6):493-505. https://doi.org/10.1056/NEJMoa1105243

12. Granich RM, Gilks CF, Dye C, De Cock KM, Williams BG. Universal voluntary HIV testing with immediate antiretroviral therapy as a strategy for elimination of HIV transmission: a mathematical model. Lancet. 2009;373(9657):48-57. https://doi.org/10.1016/S0140-6736(08)61697-9

13. Smith LR, Patterson TL, Magis-Rodriguez C, Ojeda VD, Burgos JL, Rojas SA, et al. Engagement in the HIV care continuum among key populations in Tijuana, Mexico. AIDS Behav. 2016;20(5):1017-25. https://doi.org/:10.1007/s10461-015-1186-8

14. Silva CE, Smith LR, Patterson TL, Semple SJ, Harvey-Vera A, Nunes S, et al. Stigma and web-based sex seeking among men who have sex with men and transgender women in Tijuana, Mexico: a cross-sectional study. JMIR Public Health. 2020;6(1);e14803. https://doi.org/10.2196/14803.

15. Semple SJ, Pines HA, Strathdee SA, Harvey-Vera A, Rangel G, Magis-Rodriguez C, et al. Uptake of a partner notification model for HIV among men who have sex with men and transgender women in Tijuana, Mexico. AIDS Behav. 2018;22(7):2042-55. https://doi.org/10.1007/s10461-017-1984-2

16. Sedia Biosciences Corporation. Sedia ${ }^{\mathrm{TM}}$ HIV-1 LAg-Avidity EIA Package Insert. Portland, OR; 2016 [cited 2018 Oct 6]. Available from: http://www.sediabio.com/products/lag-avidity-eia

17. Chaillon A, Gianella S, Wertheim JO, Richman DD, Mehta SR, Smith DM. HIV migration between blood and cerebrospinal fluid or semen over time. J Infect Dis. 2014;209(10):1642-52. https://doi.org/10.1093/infdis/jit678

18. Smith DM, May SJ Tweeten S, Drumright L, Pacold ME, Kosakovsky-Pond SL, et al. A public health model for the molecular surveillance of HIV transmission in San Diego, California. AIDS. 2009;23(2):225-32. https://doi.org/10.1097/QAD.0b013e32831d2a81

19. Ragonnet-Cronin ML, Shilaih M, Günthard HF, Hodcroft EB, Boni J, Fearnhill E, et al. A direct comparison of two densely sampled epidemic: the UK and Switzerland. Sci Rep. 2016;6:32251. https://doi.org/ 
20. Patrick R, Jain J, Harvey-Vera A, Semple J, Rangel G, Patterson TL, et al. Perceived barriers to pre-exposure prophylaxis use among HIV-negative men who have sex with men in Tijuana, Mexico: a latent class analysis. PLoS One. 2019;14(8):e0221558. https://doi.org/10.1371/journal.pone.0221558

21. Herek GM, Cogan JC, Gillis JR, Glunt EK. Correlates of internalized homophobia in a community sample of lesbians and gay men. J Gay Lesbian Med Assoc. 1998;2(1):17-26.

22. Centers for Disease Control and Prevention. HIV/STD risks in young men who have sex with men who do not disclose their sexual orientation--six US cities, 1994-2000. MMWR Morb Mortal WKLY Rep. 2003;52(5):81-6.

23. Moser A, Stuck AE, Silliman RA, Ganz PA, Clough-Gorr KM. The eight-item modified Medical Outcomes Study Social Support Survey: psychometric evaluation showed excellent performance. J Clin Epidemiol. 2012;65(10):1107-16. https://doi.org/10.1016/j.jclinepi.2012.04.007

24. Larsson A. AliView: a fast and lightweight alignment viewer and editor for large datasets. Bioinformatics. 2014;30(22):3276-8. https://doi.org/10.1093/bioinformatics/btu531

25. Kosakovsky Pond SL, Weaver S, Leigh Brown AJ, Wertheim JO. HIV-TRACE (Transmission Cluster Engine): a tool for large scale molecular epidemiology of HIV-1 and other rapidly evolving pathogens. Mol Biol Evol. 2018;35(7):1812-9. https://doi.org;10.1093/molbev/msy016

26. Wertheim JO, Kosakovsky Pond SL, Forgione LA, Mehta SR, Murrell B, Shah S, et al. Social and genetic networks of HIV-1 transmission in New York City. PLoS Pathog. 2017;13(1):e1006000. https://doi.org/10.1371/journal.ppat.1006000

27. Oster AM, Wertheim JO, Hernandez AL, Ocfemia MCB, Saduvala N, Hall HI. Using molecular HIV surveillance data to understand transmission between subpopulations in the United States. J Acquir Immune Defic Syndr. 2015;70(4):444-51. https://doi.org/10.1097/QAI.0000000000000809

28. Oster AM, France AM, Panneer N, Bañez Ocfemia MC, Campbell E, Dasgupta S, et al. Identifying clusters of recent and rapid HIV transmission through analysis of molecular surveillance data. J Acquir Immune Defic Syndr. 2018;79(5):543-50. https://doi.org/10.1097/QAI.0000000000001856

29. Little SJ, Kosakovsky Pond SL, Anderson CM, Young JA, Wertheim JO, Mehta SR, et al. Using HIV networks to inform real time prevention interventions. PLoS One. 2014;9(6):e98443. https://doi.org/10.1371/journal.pone.0098443

30. Wertheim JO, Oster AM, Hernandez AL, Saduvala N, Ocfemia MCB, Hall HI. The international dimension of the U.S. HIV transmission network and onward transmission of HIV recently imported into the United States. AIDS Res Hum Retroviruses. 2016;32(10-11):1046-53. https://doi.org/10.1089/AID.2015.0272

31. Whiteside YO, Song R, Wertheim JO, Oster AM. Molecular analysis allows inference into HIV transmission among young men who have sex with men in the United States. AIDS. 2015;29(18):2517-22. https://doi.org10.1097/QAD.0000000000000852

32. Morgan E, Skaathun B, Schneider JA. Sexual, social, and genetic network Oveorlap: a socio-molecular approach toward public health intervention of HIV. Am J Public Health. 2018;108(11):1528-34. https://doi.org/10.2105/AJPH.2018.304438

33. Westreich D, Greenland S. The table 2 fallacy: presenting and interpreting confounder and modifier coefficients. Am J Epidemiol. 2013;177(4):292-8. https://doi.org/10.1093/aje/kws412

34. Newman M, Barabasi Al, Watts D, Watts DJ. The structure and dynamics of networks. Princeton, NJ: Princeton University Press; 2006.

35. Anguiano J. Repression and resistance: a social history of the gay social movement of Tijuana, México 1980-1993. Kalamazoo, MI: Western Michigan University; 2019.

36. Mimiaga MJ, Noonan E, Donnell D, Safren SA, Koenen KC, Gortmaker S, et al. Childhood sexual abuse is highly associated with HIV risk-taking behavior and infection among MSM in the EXPLORE Study. J Acquir Immune Defic Syndr. 2009;51(3):340-8. https://doi.org/10.1097/QAI.0b013e3181a24b38

37. Welles SL, Baker AC, Miner MH, Brennan DJ, Jacoby S, Rosser BRS. History of childhood sexual abuse and unsafe anal intercourse in a 6-city study of HIV-positive men who have sex with men. 2009;99(6):1079-86. https://doi.org/10.2105/AJPH.2007.133280 
38. Pines HA, Goodman-Meza D, Pitpitan EV, Torres K, Semple SJ, Patterson TL. HIV testing among men who have sex with men in Tijuana, Mexico: a cross-sectional study. BMJ Open. 2016;6(2):e010388. https://doi.org/10.1136/bmjopen-2015-010388

Funding: National Institutes of Health (NIH) grant NIDA R01 DA037811 (TLP), NIDA K01 DA040543 (HAP), AI135992 (JP), the San Diego Center for AIDS Research (SD CFAR) Next Generation grant P30 AI036214 (BS), SD CFAR T32 P30 AI036214 (BS), and NIDA K01 DA049665 (BS).

Authors' Contribution: Design and implementation of the research and results analysis:BS, HAP, TLP, SJS, JP, SRM. Manuscript drafting: BS. Manuscript review: SJS, AHV, GR.

Acknowledgements: To Montserrat Galindo Moreno, Monica Fabiola Zapién Vásquez, Sergio Alfonso Rivera Flores, Ebermhi Federico García Hernández, Karla Farfán Martínez, for data collection, and to Enlaces investigators Steffanie Strathdee, Davey Smith, and Laramie Smith, for consenting to the use of the data.

Conflict of Interests: The authors declare no conflict of interest. 